\title{
SISTEMATIZAÇÃO DA ASSISTÊNCIA DE ENFERMAGEM EM UMA UNIDADE PSIQUIÁTRICA: RELATO DE EXPERIÊNCIA
}

\section{Luiza Fortes Lamberty'; Munah Najeh Saleh Ahmad Maruf ${ }^{2}$; Ana Luiza Rios Antunes $^{3}$; Eduarda Rodrigues Machado ${ }^{4}$; Flávia Dorneles Saleh ${ }^{5}$; João Victor Quartieri Simões Pires ${ }^{6}$; Morgana Aline da Silva ${ }^{7}$; Dirce Stain Backes ${ }^{8}$}

\section{RESUMO}

Objetivo: Relatar a experiência vivenciada na psiquiatria com enfoque no processo de enfermagem. Metodologia: Trata-se de um relato de experiência realizado no decorrer da disciplina de Fundamentos Teóricos e Filosóficos de enfermagem, no segundo semestre de 2019. Realizado na unidade psiquiátrica de uma instituição de saúde, localizada na região central do Rio Grande do Sul, a partir de visitas in loco. Resultados e Discussões: Observou-se que a utilização do processo de enfermagem atribui em melhoras significativas no momento de realizar os diagnósticos de enfermagem, bem como na melhora da saúde deste indivíduo. Conclusão: Conclui-se que as atividades realizadas foram de suma importância para a promoção da saúde, onde foi possível refletir escrupulosamente acerca da taxonomia NANDA (2018-2020) e do processo de trabalho na organização e na SAE, fundamentada na teoria da complexidade.

Palavras-chave: Cuidado; Processo de Trabalho; Saúde Mental. Eixo Temático: Atenção Integral e Promoção à Saúde.

\footnotetext{
${ }^{1}$ Acadêmica do Curso de Enfermagem. Universidade Franciscana - UFN. Email: luiza.lamberty@gmail.com

${ }^{2}$ Acadêmica do Curso de Enfermagem. Universidade Franciscana - UFN. Email: munahsaleh1999@gmail.com

${ }^{3}$ Acadêmica do Curso de Enfermagem. Universidade Franciscana - UFN. Email: riosana1306@gmail.com

${ }^{4}$ Acadêmica do Curso de Enfermagem. Universidade Franciscana - UFN. Email: eduardamachado886@gmail.com

${ }^{5}$ Acadêmica do Curso de Enfermagem. Universidade Franciscana - UFN. Email: flaviasaleh222@gmail.com

${ }^{6}$ Acadêmico do Curso de Enfermagem. Universidade Franciscana - UFN. Email: quarti.joao@gmail.com

${ }^{7}$ Acadêmica do Curso de Enfermagem. Universidade Franciscana - UFN. Email: morgana.a.dasilva@gmail.com

${ }^{8}$ Doutora em Enfermagem. Docente do Curso de Graduação em Enfermagem e Coordenadora do Mestrado Profissional em Saúde Materno Infantil da Universidade Franciscana - UFN. Email: backesdirce@ufn.edu.br
} 


\section{INTRODUÇÃO}

A Sistematização da Assistência de enfermagem, também chamada de processo de enfermagem, é composta por cinco etapas, classificadas de coleta de dados, diagnóstico de enfermagem, planejamento, intervenções e avaliação dos resultados esperados (POTTER, 2009).

Regulamenta no Brasil como um método que organiza o trabalho profissional, possibilitando a implementação do Processo de Enfermagem (PE), instrumento metodológico que orienta o cuidado profissional de enfermagem. $\mathrm{O}$ enfermeiro, com estas atribuições tem o dever de diagnosticar, prescrever os cuidados e tratar este paciente, através da singularidade de cada indivíduo (SILVA; GARANHANI; PERES, 2015).

A utilização deste processo de enfermagem, atribui diretamente na melhora da saúde do paciente, visto que é uma metodologia criada como um instrumento auxiliar. Quando seguido, o trabalho do enfermeiro fica devidamente realizado e com os resultados satisfatórios esperados, trazendo melhorias ao cuidado, serviço, ambiente na convivência das equipes (SANTOS; MONTEZELI; PERES, 2012).

Pode-se associar a utilização deste processo com a teoria da complexidade, onde tem como pauta inicial a organização dos pensamentos, e assim uma maior efetividade no momento de realização da SAE. Tendo em vista que no pensamento complexo se avalia a situação e pensado de forma que quando utilizado, pode-se obter melhora significativa na qualificação do gerenciamento, bem como no planejamento de suas atividades, melhorando assim as práticas de promoção à saúde (SILVA et al. 2015).

Entre as etapas, a coleta de dados, podendo ser chamado como o histórico de enfermagem, no qual se dispõe todo o processo sistemático e contínuo, realizado por diversas técnicas e métodos dimensional. Com intuito de colher dados, visando o indivíduo como um todo, juntamente a sua família e seu estado social, ambiental e 
psicossocial. O objetivo é obter informações no seu processo de saúde-doença, para que possa então realizar os diagnósticos de enfermagem de forma precisa (MARINELLI; SILVA; SILVA, 2015).

No diagnóstico de enfermagem, é feito o agrupamento de todos os dados coletados do paciente, para que assim o enfermeiro possa tomar alguma decisão diante a ele, constituindo um dos aspectos mais importante para o planejamento, relacionando com a tomada de decisão até resultar nos dados esperados com esse diagnóstico (MARINELLI; SILVA; SILVA, 2015).

Relacionados com os diagnósticos de enfermagem, originamos 0 planejamento desta intervenção, onde é relacionado diretamente com os dados que o enfermeiro obteve através da coleta de dados com o paciente, junto a um plano de ação, ou intervenção que será realizada após este planejamento. A implementação é a ação ou intervenção direcionada do planejamento de enfermagem, através dela obtém-se os dados e resultados deste paciente (SILVA. et al, 2011).

Com tudo, a avaliação de enfermagem é contínua e deliberada, pois nela pode-se verificar as respostas e mudanças que ocorrem neste paciente após realizada a implementação. Onde se observa os resultados esperados, ou a necessidade de mudanças e adaptação no processo de enfermagem. Esta Sistematização deve respeitar as condições humanas, que por vez podem ser de baixa, média ou alta complexidade (SOARES et al, 2015).

\section{METODOLOGIA}

A respectiva ação ocorreu ao decorrer do segundo semestre do ano de 2019, na disciplina de Fundamentos Teórico Filosóficos da Enfermagem. Este relato de experiência ocorreu em uma instituição de saúde na região central do Rio Grande do Sul, em uma unidade psiquiátrica. Sucedeu-se através de uma visita in loco onde foi realizado uma entrevista. Alem disso, é um trabalho de natureza teórico-reflexivo, cuja fundamentação baseia-se na formulação discursiva acerca da temática, consultado em materiais acadêmicos científicos. 


\section{RESULTADOS E DISCUSSÕES}

Através das entrevistas e da realização da SAE em um determinado paciente, através da coleta de todas as etapas, foram elencados os seguintes diagnósticos de enfermagem:

\section{Coleta de dados:}

E.S refere ter internado inicialmente por depressão, pois sentia que estava cansada fisicamente e espiritualmente. $\mathrm{Na}$ entrevista apresentou-se calma e sem sinais evidentes de transtornos. A paciente contou-nos sua história e diz que já havia sido internada mais de quinze vezes, todas elas pelo mesmo motivo. Conta ter dois filhos, com uma diferença de idade de 10 anos entre eles, e que apresentou dificuldades e complicações durante as duas gestações. Refere não ter uma relação muito boa com o mais velho, o que a deixa consternada.

Refere já ter passado por dois casamentos e atualmente está namorando. Sentia-se muito estressada, perdia a paciência muito fácil e não conseguia raciocinar direito. Mora junto com o namorado, porém, não consegue mais conviver com ele, por este motivo resolveu buscar ajuda na internação. Durante as perguntas referiu ter momentos em que pensou em cometer suicídio, refere já ter tentado diversas vezes tirar sua própria vida com medicações.

\section{Diagnósticos:}

Os diagnósticos foram determinados de acordo com o caso e com a interpretação clínica e crítica, enfatizando a teoria da complexidade. Sendo assim:

- Risco de suicídio

(Domínio 11- Segurança/ proteção; Classe3 - Violência; aprovado em 2000 Revisado em 2013,2017).

Definição: suscetibilidade à lesão auto infligida que ameaça a vida.

A paciente apresenta como características definidoras para esse diagnóstico: 
Acúmulo de Medicamentos Armazenados; Mudança Acentuada de Comportamento; Recuperação Eufórica Repentina de Depressão Profunda; Perda de Relacionamento Significativo; Vida Familiar Problemática; relata desejo de morrer.

\section{Eixos:}

Foco do diagnóstico: problema; sujeito do diagnóstico; indivíduo; julgamento; situacional (história de tentativa de suicídio); localização; corpo; idade; adulto; tempo; contínuo.

- Tristeza crônica

(Domínio 9- Enfrentamento/ tolerância ao estresse; Classe 2- Resposta de enfrentamento, aprovado em 1998 - Revisado em 2017).

Definição:

Padrão cíclico, recorrente e potencialmente progressivo de tristeza disseminada, vivenciada (por pai/mãe, cuidador ou indivíduo com doença crônica ou deficiência) em resposta à perda contínua ao longo da trajetória de uma doença ou deficiência.

\section{O paciente apresenta como características definidoras para esse diagnóstico:}

Tristeza; Sensação que Interfere no Bem-Estar; Sentimentos negativos devastadores.

\section{Eixos:}

Foco do diagnóstico: problema; sujeito do diagnóstico; indivíduo; julgamento; crônico (crise no controle da doença); localização; corpo; idade; adulto; tempo; contínuo.

- Sofrimento espiritual

(Domínio 10- Princípios da vida; Classe3- coerência entre valores/ crenças/atos; aprovado em 1978- Revisado em 2002, 2013, 2017). 
Definição:

Estado de sofrimento relacionado à capacidade prejudicada de experimentar significado na vida por meio de conexões consigo mesmo, com os outros, com o mundo ou com um poder maior

$\underline{\text { O paciente apresenta como características definidoras para esse diagnóstico: }}$

Ansiedade; Questionamento do Sentido da Vida, Medo, Redução da Serenidade; Estratégias de Enfrentamento Ineficaz.

\section{Eixos:}

Foco do diagnóstico: problema; sujeito do diagnóstico; indivíduo; julgamento; situacional (depressão); localização; corpo; idade; adulto; tempo; contínuo.

- Enfrentamento familiar comprometido

(Domínio 9- Enfrentamento/tolerância ao estresse; Classe2- respostas de enfrentamento; aprovado em 1980- Revisado em 1996, 2017).

Definição:

Uma pessoa importante, geralmente apoiadora (membro da família, parceiro ou amigo próximo), oferece apoio, conforto, assistência ou encorajamento insuficiente, ineficaz ou comprometido, que pode ser necessário ao paciente para administrar ou dominar as tarefas adaptativas relacionadas a seu desafio de saúde.

$\underline{O}$ paciente apresenta como característica definidoras para esse diagnóstico:

Limitação na Comunicação Entre a Pessoa de Apoio e o Paciente; Pessoa de Apoio Afasta-se do Paciente; Comportamento Protetor da Pessoa de Apoio Incoerente com a necessidade de autonomia do paciente.

- Manutenção ineficaz da saúde 
(Domínio 1- Promoção da saúde; Classe2- Controle da saúde; aprovado em 1982Revisado em 2017).

Definição: Incapacidade de identificar, controlar e/ou buscar ajuda para manter o bem-estar.

O paciente apresenta como característica definidoras para esse diagnóstico: Sofrimento Espiritual; Tomada de Decisões Prejudicada; Recursos Insuficiente; Habilidade de comunicação ineficaz.

\section{Planejamento}

No planejamento, a paciente solicitou que adoraria realizar um passeio pelo Shopping com seu filho, ir ao cinema e comer um pastel. A ação seria realizada na visita seguinte, porém, foi informado que ela teria dado alta ao decorrer da semana.

\section{Intervenção}

Ao desenvolver a intervenção ressaltou-se a realização de ações voltadas para promoção e manutenção da saúde mental, englobando estratégias frente a melhora do humor, fortalecimento do convívio familiar, redução do sofrimento psíquico e restauração da saúde mental.

Como as ações planejadas não foram executadas, os relatores destacam a importância de trabalhar as ações voltadas a manutenção e promoção da saúde mental no âmbito de prevenção e na assistência primária a saúde, evitando que usuários como a E.S enfrente uma hospitalização psiquiátrica.

Ações de saúde mental no âmbito da atenção primária devem ser fundamentadas nos princípios do SUS e da reforma psiquiátrica, cujo principal objetivo é a desinstitucionalização, o que pressupõe a manutenção e seguimento do doente mental em seu território, possibilitando a preservação dos vínculos com estes pacientes e suas famílias evitando assim, internações desnecessárias e preferindo esta opção quando se trata de emergências psiquiátricas (NASCIMENTO et al., 2021). 
Desta forma, a escuta destes profissionais é uma tecnologia leve, de relevância no âmbito da saúde mental, para que o indivíduo expresse os diversos sentimentos que integram sua rotina de vida, e que podem gerar ou agravar o sofrimento mental tanto ao nível individual quanto coletivo (NASCIMENTO et al., 2017).

Embora a promoção da saúde e a da saúde mental sejam abor dagens complementares, a promoção da saúde em geral foca nos fatores relacionados ao estilo de vida (promoção de atividade física, hábitos de vida saudáveis, redução do uso de substâncias), enquanto a promoção da saúde mental objetiva aumentar diretamente a resiliência e empoderar as pessoas para lidarem melhor com as adversidades ou eventos estressantes, focando nas habilidades cognitivas, sociais e emocionais, utilizando, principalmente, estratégias baseadas na técnica de solução de problemas, treinamento de habilidades sociais e apoio social (SOUZA et al., 2018)

\section{$\underline{\text { Resultados }}$}

Como obtenção de resultados, analisamos que caso a ação tivesse ocorrido, a paciente conseguiria ter momentos de alegria ao estar junto do filho e com isso alcançaria uma melhora no estado clínico, principalmente emocional.

\section{CONCLUSÃO}

Percebeu-se que as atividades realizadas foram de suma importância para a promoção da saúde, onde foi possível refletir escrupulosamente acerca da taxonomia NANDA (2018-2020) e da utilidade prática na organização da SAE. Conclui-se que a atividade de promoção e prevenção da saúde relacionadas aos cuidados ao paciente, viabiliza que o cuidado em saúde vai muito além das dimensões técnicas. 
Além disso, o cuidado está no acolhimento, na comunicação terapêutica, em um gesto ou através de olhar. O cuidado ampliado em saúde é importante tanto para a vida acadêmica, quanto à profissional, pois é preciso olhar o ser humano como um todo, enxergando sua singularidade e não somente pelo viés de seu processo de doença.

\section{REFERÊNCIAS}

COFEN, RESOLUÇÃO COFEN-358/2009. Disponível em:

https://enfermagem.jatai.ufg.br/up/194/o/Resolu\%C3\%A7\%C3\%A30 n\%C2\%BA3582009.pdf

Diagnósticos de enfermagem de Nanda: definições e classificação 2018-2020. 11.ed. Porto Alegre: Artmed, 2018. Disponível em: http://nascecme.com.br/2014/wpcontent/uploads/2018/08/NANDA-I-2018 2020.pdf

MARINELLI, N. P. SILVA, A. R. A. SILVA, D. N. O. Sistematização da assistência de enfermagem: desafios para a implantação. Rev. Enferm Contemp. 2015; v. 4, n. Disponível em:

https://webcache.googleusercontent.com/search?q=cache:mv1 5sCfafMJ:https://ww w5.bahiana.edu.br/index.php/enfermagem/article/download $/ 523 / 553+\& \mathrm{~cd}=3 \& \mathrm{hl}=$ pt$\underline{B R \& c t=c l n k \& g l=b r}$

NASCIMENTO M.G.G, et al. O processo de trabalho do enfermeiro na promoção da saúde mental da população atendida na ESF: uma análise reflexiva. Revista de Enfermagem do Centro-Oeste Mineiro, 2017;7: Disponível em:

http://dx.doi.org/10.19175/recom.v7i0.2097

NASCIMENTO, A.H. et al., Relato de experiência acerca do cuidado integral de portadores de sofrimento psíquico na atenção primária de saúde. Revista

Eletrônica Acervo Saúde, vol. 13 (5). 1-6. DOI:

https://doi.org/10.25248/REAS.e7105.2021 
POTTER PERRY. Fundamentos de enfermagem, $7^{7 \underline{a} e d . ~ 2009 . ~ D i s p o n i ́ v e l ~ e m: ~}$ https://sibiuepa.files.wordpress.com/2017/07/fundamentos-de-enfermagem-potter.pdf

SANTOS, J. O. F, MONTEZELI, J. H, PERES, A. M. Autonomia profissional e sistematização da assistência de enfermagem: percepção de enfermeiros. Minas Gerais. Rev. Min. Enferm. 2012, v. 16, n. 2. Disponível em http://www.reme.org.br/artigo/detalhes/526.

SILVA, E. G. C. et al. O conhecimento do enfermeiro sobre a sistematização da assistência de enfermagem: da teoria à prática. Rev Esc Enferm USP. 2011, v, 45. n, 6. Disponível em: http://www.scielo.br/pdf/reeusp/v45n6/v45n6a15.pdf

SILVA, P. J. et al. Sistematização da assistência de enfermagem na graduação: um olhar sob o pensamento complexo. Rev. Latino-Am. Enfermagem. 2015, v.21, n. 1. Disponível em: http://www.scielo.br/pdf/rlae/v23n1/pt 0104-1169-rlae-23-0100059.pdf

SILVA, P. S; GARANHANI, M. L; PERES, A. M. Sistematização da assistência de enfermagem na graduação: um olhar sob o pensamento complexo. Rev. Latino-Am. Enfermagem. 2015, v. 1, n. 4. Disponível em:

https://www.scielo.br/j/rlae/a/4mwpmn7m7KY7Y76XnRnCsyK/?format=pdf\&lang=pt

SOARES, M, I. et al. Sistematização da assistência de enfermagem: facilidades e desafios do enfermeiro na gerência da assistência. Rev Escola Anna Nery. 2015, v. 19, n. 1. Disponível em:

https://www.scielo.br/j/ean/a/ghYPrPYCdg68TBW5yxrGqba/?lang=pt\#ModalArticles

SOUZA J. et al., Promotion of women's mental health: the influence of physical health and the environment. Rev Bras Enferm. 2019;72(Suppl 3):184-90. doi: http://dx.doi.org/10.1590/0034-7167-2018-0415 